\title{
Prolactin Levels in Schizophrenic Patients Receiving Perospirone in Comparison to Risperidone
}

\author{
Takashi Togo ${ }^{1,2, *}$, Eizo Iseki ${ }^{2}$, Mika Shoji ${ }^{1}$, Ikuo Oyama ${ }^{1}$, Akihiko Kase ${ }^{1}$, Hirotake Uchikado ${ }^{2}$, \\ Omi Katsuse $^{2}$ and Kenji Kosaka ${ }^{2}$ \\ ${ }^{1}$ Yokohama Maioka Hospital, Yokohama 244-0813, Japan \\ ${ }^{2}$ Department of Psychiatry, Yokohama City University School of Medicine, Yokohama 236-0004, Japan
}

Received November 21, 2002; Accepted January 9, 2003

\begin{abstract}
Serum prolactin levels were investigated in 41 patients with schizophrenia who were receiving clinically effective doses of perospirone or risperidone for more than 4 weeks. In order to determine baseline prolactin levels, blood samples were obtained in the morning, $10-14 \mathrm{~h}$ after antipsychotic medication. Median levels were within normal limits in both female and male patients receiving perospirone, while risperidone induced significant elevation. These results suggest that in contrast to risperidone, where baseline prolactin levels were elevated 5.3-fold in female and 4.2-fold in male patients, baseline prolactin levels are not elevated after treatment with perospirone. However, these results should be cautiously interpreted, because drug-by-time interaction has previously been reported in antipsychotic-induced hyperprolactinemia.
\end{abstract}

Keywords: prolactin, perospirone, schizophrenia

Hyperprolactinemia is a common side effect that may arise from the use of antipsychotic agents $(1,2)$. Before the introduction of clozapine, it was assumed that all antipsychotics cause extrapyramidal side effects and elevate prolactin levels by antagonizing dopamine $\mathrm{D}_{2}$ receptors. In contrast, the new generation of so-called atypical antipsychotics, which combine serotonin (5hydroxytryptamine-2 $\left.\left[5-\mathrm{HT}_{2}\right]\right) / \mathrm{D}_{2}$ receptors, have variable tendencies to induce hyperprolactinemia (3).

Perospirone is a recently developed antipsychotic agent for the treatment of schizophrenia (for review, see refs. 4, 5). Its primary mode of action is antagonism of 5- $\mathrm{HT}_{2 \mathrm{~A}}$ and dopamine $\mathrm{D}_{2}$ receptors, with a profile similar to that of risperidone. Similar to other atypical antipsychotic agents and in contrast to haloperidol, perospirone is effective against not only positive symptoms of schizophrenia, but also against negative symptoms such as blunted affect, social withdrawal, and difficulty in abstract thinking (6). Extrapyramidal symptoms occur less often and are milder with perospirone than with haloperidol or mosapramine $(6,7)$. However, because perospirone has been recently introduced and used clinically only in Japan, antipsychotic efficacy as

*Corresponding author (affiliation \#1). FAX: +81-45-823-9757

E-mail: togo-t@rd6.so-net.ne.jp well as the adverse effects of perospirone have not been fully investigated. In the present study, we have investigated the baseline serum prolactin levels in patients treated with clinically effective doses of perospirone in comparison to risperidone. This is the first report of serum prolactin levels in schizophrenic patients treated with perospirone.

This study was performed at the Yokohama Maioka Hospital, Japan, and all patients were of Japanese decent. Patients with a diagnosis of schizophrenia were included if they were receiving either perospirone or risperidone and if they had been receiving a stable dose of these agents for more than 4 weeks. Patients were excluded from participation if they had been receiving other antipsychotics, or if they were receiving any medication or had any physical condition that could affect prolactin levels. In compliance with therapy-concurrent naturalistic study design, other concomitant medications including antiparkinsonian or benzodiazepines that are not known to affect prolactin level were permitted. The last antipsychotic medication was given $10-14 \mathrm{~h}$ before blood withdrawal, and all blood samples were obtained between 8:00 AM and 10:00 AM. Samples were shipped to BML, Inc. (Tokyo) for assay. Prolactin levels were determined by an immunoradiometric assay with the 
WHO standard kit. The upper normal limits of the levels in females and males are 14.6 and $9.7 \mathrm{ng} / \mathrm{ml}$, respectively (BML, Inc.). This study was approved by the Human Subjects Review Committee of the Yokohama Maioka Hospital. For the present study, each patient provided written informed consent for participation after the procedures had been fully explained. Data was analyzed using Sigma Stat for Windows version 2.03 (SPSS Science, Chicago, IL, USA), and significance levels were set as $P<0.05$.

The results of this study are shown in Fig. 1 ( $a$ and $b$ ) and summarized in the Table 1. Although there was a significant difference for ages among the different gender and treatment groups $(P<0.05$, one-way
ANOVA), there were no significant differences found between those treated with perospirone or with risperidone, in either female or male patients $(P=0.82$ and 0.71 , respectively, $t$-test). Based on the facts that $1 \mathrm{mg}$ risperidone is equivalent to $2 \mathrm{mg}$ of haloperidol (3) and that $4 \mathrm{mg}$ perospirone is equivalent to $1 \mathrm{mg}$ of haloperidol (8), a daily dose of $1 \mathrm{mg}$ risperidone is considered clinically equivalent to $8 \mathrm{mg}$ of perospirone. There were no significant differences in either the dose of medication, after conversion of the risperidone dose to perospirone equivalents $(P=0.25$, Kruskal-Wallis one-way ANOVA of ranks), or in the duration of treatment ( $P=0.07$, Kruskal-Wallis one-way ANOVA of ranks).

The median serum prolactin levels in female and

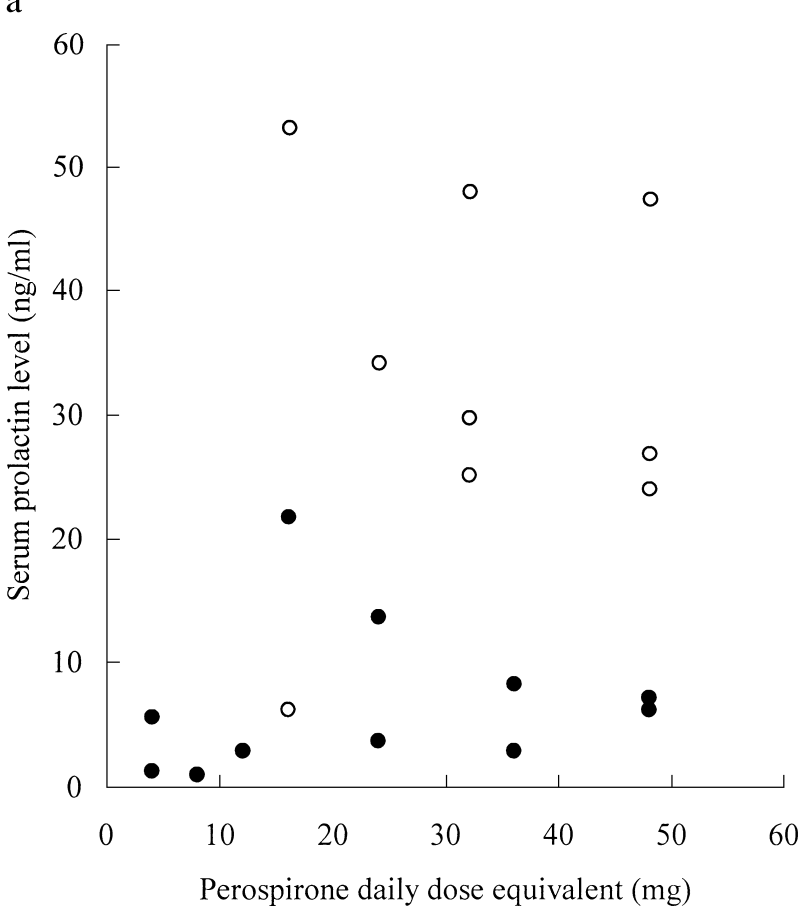

b

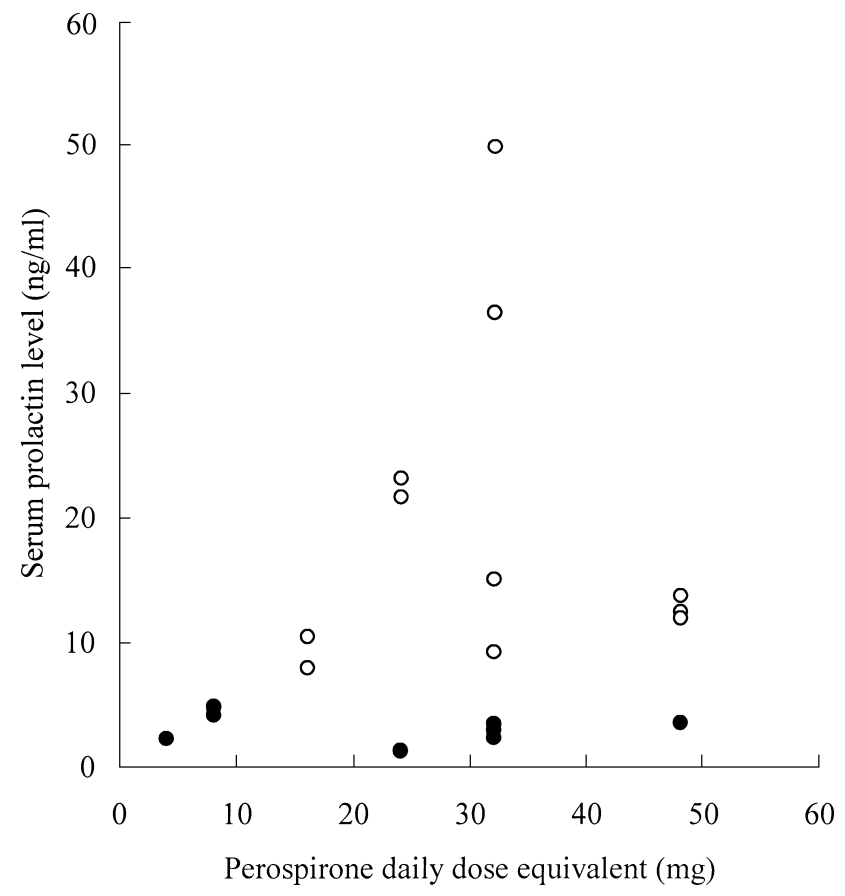

Fig. 1. Serum prolactin levels in schizophrenic patients treated with perospirone or risperidone. a: Serum prolactin levels in female patients treated with perospirone (filled circles) or risperidone (open circles). A dose of $1 \mathrm{mg}$ of risperidone was considered the equivalent of $8 \mathrm{mg}$ of perospirone $(3,8)$. b: Serum prolactin levels in male patients treated with perospirone (filled circles) and risperidone (open circles).

Table 1. Baseline serum prolactin levels in each treatment group $10-14 \mathrm{~h}$ after drug intake

\begin{tabular}{llccccc}
\hline \multicolumn{2}{l}{ Treatment group } & $\mathrm{N}$ & Age & Dose $(\mathrm{mg})$ & Duration (weeks) & Prolactin $(\mathrm{ng} / \mathrm{ml})$ \\
\hline \multirow{2}{*}{ Perospirone } & female & 11 & $54.1 \pm 10.8$ & $24(4-48)$ & $28(6-60)$ & $5.6(1.0-21.8)$ \\
& male & 10 & $45.1 \pm 11.8$ & $24(4-48)$ & $17.5(4-56)$ & $3.25(1.3-4.9)$ \\
\multirow{3}{*}{ Risperidone } & female & 9 & $55.1 \pm 9.1$ & $4(2-6)$ & $66(4-216)$ & $29.8^{*}(6.2-53.2)$ \\
& male & 11 & $43.2 \pm 11.8$ & $4(2-6)$ & $75(4-271)$ & $13.8^{*}(8.0-49.9)$ \\
\hline
\end{tabular}

Ages are reported as the mean \pm S.D.; dose, duration and serum prolactin levels are reported as the median and range. The upper normal limits of prolactin level in females and males are 14.6 and $9.7 \mathrm{ng} / \mathrm{ml}$, respectively. *Significantly different compared to the patients receiving perospirone in both female and male patients $(P<0.001)$. 
male patients treated with perospirone were 5.6 and $3.25 \mathrm{ng} / \mathrm{ml}$, respectively, while those treated with risperidone were 29.8 and $13.8 \mathrm{ng} / \mathrm{ml}$, respectively. There were significant differences between the prolactin levels in patients treated with perospirone or risperidone, both in female and male patients $(P<0.001$, Mann-Whitney rank sum test). Prolactin levels in 10 of a total of 11 female and in all 10 male patients treated with perospirone were below the upper normal limit, while the levels in 8 of the 9 female and in 9 of the 11 male patients treated with risperidone were above this limit. Gender differences in prolactin levels were found in patients treated with risperidone $(P<0.05$, MannWhitney rank sum test). Despite a higher tendency in female patients, the difference in prolactin levels in patients treated with perospirone did not reach significance $(P=0.16$, Mann-Whitney rank sum test).

The frequency and magnitude of hyperprolactinemia induced by perospirone have been reported only in clinical trials before being available for use in the clinic. In a phase I trial, administration of low doses $(1-8 \mathrm{mg})$ in 8 healthy volunteers resulted in dose-dependent acute elevation of prolactin plasma levels, while baseline levels were unchanged (9). The levels reached a peak level $1-2 \mathrm{~h}$ after administration, with twice the peak level in subjects receiving $8 \mathrm{mg}$ of perospirone compared to those receiving $2 \mathrm{mg}$ haloperidol. In phase III trials, the frequency of hyperprolactinemia was reported to be $30-37 \%$ in patients treated with perospirone, which is comparable to that with haloperidol and less frequent than that with mosapramine $(7,10)$. However, the magnitude of the levels was not described, and drug-by-time interaction was not considered in these trials. In phase I, II, and III trials of perospirone, no adverse effects were associated with hyperprolactinemia (such as amenorrhoea, galactorrhea, or sexual dysfunction).

In the present study, we investigated baseline serum prolactin levels in patients receiving clinically effective doses of perospirone in comparison to those receiving risperidone. These two agents are known to have a similar profile of action at $5-\mathrm{HT}_{2 \mathrm{~A}}$ and dopamine $\mathrm{D}_{2}$ receptors. The median baseline prolactin levels were within normal limits both in female and male patients receiving perospirone, while risperidone gave rise to elevation of prolactin levels above these limits, in line with previous reports (11). There were significant differences in prolactin levels between patients receiving perospirone and risperidone in both female and male patients. We have here demonstrated, for the first time, that baseline serum prolactin levels in schizophrenic patients treated with perospirone are not elevated. However, the results should be interpreted with caution because drug-by-time interaction has been reported previously in antipsychotic-induced hyperprolactinemia. The increase in serum prolactin levels with atypical antipsychotics including clozapine, olanzapine, quetiapine, and risperidone, is most pronounced in the first $1-5 \mathrm{~h}$ after administration $(11,12)$, even though baseline levels are not elevated for patients receiving clozapine, olanzapine, or quetiapine. Risperidone is the exception to this pattern because the baseline prolactin levels after risperidone are also elevated (11), a finding supported by the results of the present study. Our results indicate that perospirone causes a pattern of variation in serum prolactin levels similar to that of clozapine, olanzapine, and quetiapine in terms of the low baseline levels, with greater peak prolactin levels compared to those caused by haloperidol (9). This supports the view that, similar to other atypical antipsychotic agents (13), a transient dopamine $\mathrm{D}_{2}$ receptor blockade is central to the mechanism of action of perospirone. It is currently unclear whether it is the peak or baseline level of plasma prolactin that leads to hyperprolactinemia-associated side effects.

This study is limited by the small number of subjects and by the fact that most patients were of middle age and menopause in female patients was not considered. In addition, patients taking other concomitant medications that are not known to affect serum prolactin levels were included in compliance with the therapy-concurrent naturalistic study design. Nevertheless, we conclude, that in contrast to the elevated baseline prolactin levels in patients receiving risperidone, baseline prolactin levels are not elevated in schizophrenic patients receiving clinically effective doses of perospirone. Further studies are needed to evaluate drug-by-time interaction as well as hyperprolactinemia-related side effects in various age groups among subjects treated with perospirone monotherapy.

\section{References}

1 Dickson RA and Glazer WM: Neuroleptic-induced hyperprolactinemia. Schizophr Res 35, Suppl S75 - S86 (1999)

2 Rubin RT: Prolactin and schizophrenia. In Psychopharmacology: The Third Generation of Progress, Edited by Meltzer HY, pp $803-808$, Raven Press, New York (1987)

3 American Psychiatric Association: Practice guideline for the treatment of patients with schizophrenia. Am J Psychiatry 154, Suppl 4, 1 - 63 (1997)

4 de Paulis T: Perospirone (Sumitomo Pharmaceuticals). Curr Opin Investig Drugs 3, 121 - 129 (2002)

5 Onrust SV and McClellan K: Perospirone. CNS Drugs 15, 329 337 (2001)

6 Murasaki K, Koyama T, Machiyama Y, Yagi G, Kamijima K, Toru M, Ushijima S and Miura S: Clinical evaluation of a new 
antipsychotic, perospirone $\mathrm{HCl}$, on schizophrenia: a comparative double-blind study with haloperidol. Clin Eval 24, 159-205 (1997) (text in Japanese with English abstract)

7 Kudo Y, Makajima T, Saito M, Sakai T, Otsuki S, Yamawaki S, Inanaga $\mathrm{K}$, Tashiro N, Nakane $\mathrm{Y}$ and Ogura T: Clinical evaluation of a serotonin-2 and dopamine-2 receptor antagonist (SDA), perospirone $\mathrm{HCl}$ on schizophrenia: a comparative double-blind study with mosapramine HCl. Clin Eval 24, 207 - 248 (1997) (text in Japanese with English abstract)

8 Murasaki K, Koyama T, Machiyama Y, Yagi G, Kamijima K, Kudo Y, Otsuki S, Inanaga K and Miura S: Clinical evaluation of a new antipsychotic, perospirone $\mathrm{HCl}$, on schizophrenia: late-phase II study. Kiso to Rinsho 31, 2181 - 2206 (1997) (text in Japanese with English abstract)

9 Inanaga $\mathrm{K}$, Irie $\mathrm{S}$, UraeA and Urae $\mathrm{T}$ : Phase I study of
Perospirone $\mathrm{HCl}$ (SM-9018). Kiso to Rinsho 31, 2113-2157 (1997) (in Japanese)

10 Murasaki K, Yamashita I, Machiyama Y, Yagi G, Kamijima K, Kudo Y, Otsuki S, Inanaga K and Miura S: Early phase 2 study of a new antipsychotic, perospirone HCL (SM-9018), on schizophrenia. Kiso to Rinsho 31, 2159 - 2179 (1997) (text in Japanese with English abstract)

11 Turrone P, Kapur S, Seeman MV and Flint AJ: Elevation of prolactin levels by atypical antipsychotics. Am J Psychiatry 159, 133 - 135 (2002)

12 Alexiadis M, Whitehorn D, Woodley H and Kopala L: Prolactin elevation with quetiapine. Am J Psychiatry 159, 1608-1609 (2002)

13 Seeman P: Atypical antipsychotics: mechanism of action. Can J Psychiatry 47, $27-38$ (2002) 